Moroccan J. of Pure and Appl. Anal. (MJPAA)

Volume 5(1), 2019, Pages 22-30

ISSN: Online 2351-8227 - Print 2605-6364

DOI 10.2478/mjpaa-2019-0002

\title{
Strongly Lipschitz up-Nuclear Operators
}

\author{
Amar Belacel ${ }^{1, a}$ AND Khedidja Bey ${ }^{1, b}$
}

AвSTRACT. In this paper, we introduce the notion of strongly Lipschitz up-nuclear operators. Among other results, we prove an analog of the factorization theorem for these classes and characterize their conjugates. 2010 Mathematics Subject Classification. 46A32, 47B10, 47L22.

Key words and phrases. Lipschitz operators; strongly Lipschitz p-summing; p-nuclear operators; Strongly Lipschitz up-nuclear; Strongly Lipschitz p-nuclear; Factorization Theorem.

\section{Introduction}

\section{Introduction}

The concept of nuclear operators goes back to Grothendieck early fifties. later on, it was generalized to $p$ nuclear operators $(1 \leq p<\infty)$ by Pietsch [15]. Several authors, [7, 9, 10, 11, 12, 15], have developed many properties concerning this notion especially Persson and Rienov [14, 16].

In 2012, Chen and Zheng, [4], generalized the concept of $p$-nuclear operators to Lipschitz situation, where the domain of such operators is a metric space that need not be a normed space, they published "Lipschitz $p$-integral operators and Lipschitz $p$-nuclear operators". Belacel and Chen defined and investigated "Lipschitz $(p, r, s)$-integral operators and Lipschitz $(p, r, s)$-nuclear operators" [3].

In this paper, we introduce the concept of Lipschitz $p$-nuclear operators. By introducing and studying the class of Lipschitz $p$-nuclear operators whose linear analogue was found in [14] we gave the factorization theorem. Among other results, we prove that the transpose of strongly Lipschitz $p$-nuclear operator is $p$-nuclear and we found the same results with the strongly Lipschitz $p$-nuclear operators which are introduced and studied by Chen and Zheng in [4].

Received May 15, 2019 - Accepted June 11, 2019.

(C) The Author(s) 2017. This article is published with open access by Sidi Mohamed Ben Abdallah University.

${ }^{1}$ University of Laghouat, Laboratoire de Mathématiques Pures et Appliques, 03000 Laghouat, Algeria.

a e-mail: a.belacel@lagh-univ.dz

${ }^{b}$ e-mail:kh.bey@lagh-univ.dz. 


\section{Notations and preliminaries}

Throughout this paper, $X, Y$ will denote pointed metric spaces with a base point denoted by 0 , while $E, F$ are (real or complex) Banach spaces. We let $Z^{*}$ to be the dual of the Banach space $Z$, and $B_{Z}$ is its closed unit ball. Given a linear and continuous operator $T$ (i.e. $T \in \mathcal{L}(E, F)$ ) we let $T^{*}$ denote the adjoint operator of $T$. Lip $0(X, F)$ is the space of all bounded Lipschitz mappings on $X$ into $F$ such that $T(0)=0$ with the Lipschitz norm $\operatorname{Lip}($.$) ,$ where $\operatorname{Lip}(T)$ is the infimum of all constants $C \geq 0$ such that $\left\|T x-T x^{\prime}\right\| \leq C d\left(x, x^{\prime}\right)$ for all $x, x^{\prime} \in X$, [20]. We let $X^{\sharp}$ denote the space $\operatorname{Lip}_{0}(X, \mathbb{R}), B_{X^{\sharp}}$ is the unite ball of $X^{\sharp}$ endowed with the pointwise topology. Its well known that $X^{\sharp}$ has a predual, namely the space of Arens Elles $Æ(X)$, where $Æ(X)$ is the completion of the normed space $\left(\mathcal{M}(X),\|\cdot\|_{\mathcal{M}(X)}\right)$, where $\mathcal{M}(X)$ is the real linear space of all molecules $m$ on $X$ for more details (see $[2,20]$ ). Given $T \in \operatorname{Lip}_{0}(X, F)$, there exits a unique linear map $T_{L}: Æ(X) \longrightarrow F$ such that $T=T_{L} \circ \delta_{X}$ and $\delta_{X}: X \longrightarrow Æ(X)$ defined by $\delta_{X}(x)=m_{x 0}$ isometrically embeds $X$ in $Æ(X)$. The operator $T_{L}$ is referred as the linearization of $T$ (see [20, Theorem 2.2.4]). The Lipschitz adjoint (or dual) of $T \in \operatorname{Lip}_{0}(X, F)$ were defined by Sawashima [18] as the continuous linear operator

$$
\begin{aligned}
T^{\sharp}: \operatorname{Lip}_{0}(F) & \longrightarrow \operatorname{Lip}_{0}(X) \\
g & \longmapsto T^{\sharp}(g)=g \circ T
\end{aligned}
$$

The restriction of $T^{\sharp}$ to $F^{*}$ is called the Lipschitz transpose map of $T$ and is denoted here by $T^{t}$. The letters $p, r, q, s$ will designate elements of $[1, \infty]$, and $p^{*}$ denotes the exponent conjugate to $p$ (i.e. the one that satisfies $\left.\frac{1}{p}+\frac{1}{p^{*}}=1\right)$. The concept of Lipschitz operator ideal appeared in [1] and it represented as follows

$$
\mathcal{I}_{\text {Lip }}(X, E):=\operatorname{Lip}_{0}(X, E) \cap \mathcal{I}_{\text {Lip }},
$$

where $\mathcal{I}$ be an ideal linear $[6,15]$.

Let us recall that a Lipschitz operator $T: X \longrightarrow Y$ is Lipschitz $p$-summing [8], if there is a constant $C \geq 0$ such that for $n \in \mathbb{N}, x_{1}, \ldots, x_{n}$ and $x_{1}^{\prime}, \ldots, x_{n}^{\prime} \in X$ we have

$$
\left(\sum_{j=1}^{n}\left\|T\left(x_{j}\right)-T\left(x_{j}^{\prime}\right)\right\|^{p}\right)^{\frac{1}{p}} \leq C \sup _{f \in B_{X} \sharp}\left(\sum_{j=1}^{n}\left|f\left(x_{i}\right)-f\left(x_{j}^{\prime}\right)\right|^{p}\right)^{\frac{1}{p}}
$$

the collection of all Lipschitz $p$-summing operators is denoted by $\Pi_{p}^{L}(X, Y)$ and infimum of all $C$ by $\pi_{p}^{L}(T)$.

For a Lipschitz operator $T: X \longrightarrow F, 1<p \leq \infty$ Yahi et al. [19], introduce the class of strongly Lipschitz $p$-summing operators, if there is a Banach space $E$ and an operator $S \in \mathcal{D}_{p}(E, F)$ [i.e $S^{*} \in \Pi_{p^{*}}\left(F^{*}, E^{*}\right)$ ] such that

$$
\left|\left\langle y^{*}, T(x)-T\left(x^{\prime}\right)\right\rangle\right| \leq d\left(x, x^{\prime}\right)\left\|S^{*}\left(y^{*}\right)\right\|
$$

for all $x, x^{\prime} \in X$ and $y^{*} \in F^{*}$. The collection of all strongly Lipschitz $p$-summing operators is denoted by $\mathcal{D}_{s t, p}^{L}(X, F)$ and $d_{s t, p}^{L}(T)$ the infimum of all $d_{p}(S)$ (see [19]).

A linear operator $T: E \rightarrow F$ is $p$-nuclear $(1 \leq p \leq \infty)$, (see $[7,14])$ if and only if, $T$ can be written in the form

$$
T=\sum_{j} x_{j}^{*} \otimes y_{j}
$$

where $\left(x_{j}^{*}\right)_{j} \subset E^{*}$ and $\left(y_{j}\right)_{j} \subset F$ satisfy

$$
\left\|\left(x_{j}^{*}\right)_{j}\right\|_{p}<\infty \text { and } \sup _{\left\|y^{*}\right\| \leq 1}\left(\sum_{j}\left|\left\langle y_{j}, y^{*}\right\rangle\right|^{p^{*}}\right)^{1 / p^{*}}<\infty .
$$

Here

$$
\mathcal{N}_{p}\left(\left(x_{j}^{*}\right)_{j},\left(y_{j}\right)_{j}\right)=\left\|\left(x_{j}^{*}\right)_{j}\right\|_{p} \sup _{\left\|y^{*}\right\| \leq 1}\left(\sum_{j}\left|\left\langle y_{j}, y^{*}\right\rangle\right|^{p^{*}}\right)^{1 / p^{*}} .
$$


Moreover, $v_{p}(T)=\inf \mathcal{N}_{p}\left(\left(x_{j}^{*}\right)_{j},\left(y_{j}\right)_{j}\right)$, the infimum being taken over all such representations as above. The collection of all linear $p$-nuclear operators from $E$ to $F$ is denoted by $\mathcal{N}_{p}(E, F)$. Interchanging the roles of the sequences $\left(x_{j}\right)_{j}$ and $\left(y_{j}\right)_{j}$ one obtains in the same way another Banach space $\mathcal{N}^{p}(E, F)$ of operators [14], the norm being given by $v_{p}(T)=\inf \mathcal{N}^{p}\left(\left(x_{j}^{*}\right)_{j},\left(y_{j}\right)_{j}\right)$, where

$$
\mathcal{N}^{p}\left(\left(x_{j}^{*}\right)_{j},\left(y_{j}\right)_{j}\right)=\sup _{\|x\| \leq 1}\left(\sum_{j}\left|\left\langle x, x_{j}^{*}\right\rangle\right|^{p^{*}}\right)^{1 / p^{*}}\left\|\left(y_{j}\right)_{j}\right\|_{p} .
$$

A linear operator $T: E \rightarrow F$ is $p$-nuclear $(1 \leq p<\infty)$, if there are two operators $a \in \mathcal{L}\left(\ell_{p}, F\right), b \in \mathcal{L}\left(E, \ell_{\infty}\right)$ and a sequence $\lambda \in \ell_{p}$ such that the following diagram commutes:

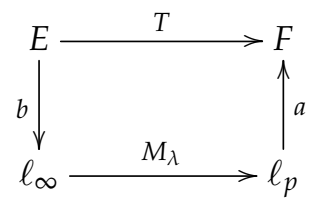

where $M_{\lambda} \in \mathcal{L}\left(\ell_{\infty}, \ell_{p}\right)$ is the diagonal operator defined as follows: $M_{\lambda}\left(\xi_{n}\right)=\left(\xi_{n} \lambda_{n}\right)_{n},\left(\xi_{n}\right)_{n} \in \ell_{\infty}$. Then $\left\|M_{\lambda}\right\|=\|\lambda\|_{p}=\pi_{p}\left(M_{\lambda}\right)$.

We set

$$
v_{p}(T)=\inf \|a\|\left\|M_{\lambda}\right\|\|b\|,
$$

the infimum being extended over all factorizations as above.

Similarly, every $T \in \mathcal{N}^{p}(E, F)$ can be factored

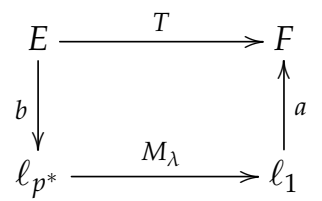

with $a, M_{\lambda}, b$ as above and

$$
\nu^{p}(T)=\inf \|a\|\left\|M_{\lambda}\right\|\|b\| .
$$

A Lipschitz operator $T: X \rightarrow F$ is strongly Lipschitz $p$-nuclear $(1 \leq p \leq \infty)$, (see [4]) if, $T$ can be written in the form

$$
T=\sum_{j} f_{j} \otimes y_{j}
$$

where $\left(f_{j}\right)_{j} \subset X^{\sharp}$ and $\left(y_{j}\right)_{j} \subset F$ satisfy

Here

$$
\left\|\left(\operatorname{Lip}\left(f_{j}\right)\right)_{j}\right\|_{p}<\infty \text { and } \sup _{\left\|y^{*}\right\| \leq 1}\left(\sum_{j}\left|\left\langle y_{j}, y^{*}\right\rangle\right|^{p^{*}}\right)^{1 / p^{*}}<\infty
$$

$$
\mathcal{N}_{p}^{L}\left(\left(f_{j}\right)_{j},\left(y_{j}\right)_{j}\right)=\left\|\left(\operatorname{Lip}\left(f_{j}\right)\right)_{j}\right\|_{p} \sup _{\left\|y^{*}\right\| \leq 1}\left(\sum_{j}\left|\left\langle y_{j}, y^{*}\right\rangle\right|^{p^{*}}\right)^{1 / p^{*}} .
$$

Moreover, $s v_{p}^{L}(T)=\inf \mathcal{N}_{p}^{L}\left(\left(f_{j}\right)_{j},\left(y_{j}\right)_{j}\right)$, the infimum being taken over all such representations as above.

A Lipschitz operator $T: X \rightarrow F$ is strongly Lipschitz $p$-nuclear $(1 \leq p \leq \infty)$, if there are two Lipschitz mappings $A \in \mathcal{L}\left(\ell_{p}, F\right), B \in \operatorname{Lip}_{0}\left(X, \ell_{\infty}\right)$ and a sequence $\lambda \in \ell_{p}$ such that the following diagram commutes:

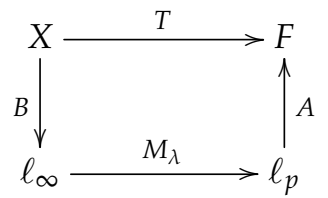


We set

$$
s v_{p}^{L}(T)=\inf \|A\|\left\|M_{\lambda}\right\| \operatorname{Lip}(B),
$$

the infimum being extended over all factorizations as above (see [4, Theorem 2.2]).

\section{Strongly Lipschitz up-nuclear operators}

In this section, we start by giving a new concept, the Lipschitz version of $N^{p}$-nuclear operator which are introduced in $[14,16]$.

Definition 4.1. Let $1<p \leq \infty$, and $T \in \operatorname{Lip}_{0}(X, F)$. $T$ is Lipschitz up-nuclear operator, if $T$ can be written in the following form

$$
T=\sum_{j} f_{j} \otimes y_{j}
$$

such that $\left(f_{j}\right)_{j} \subset X^{\sharp}$ where $X^{\sharp}$ is the space of all real-valued Lipschitz functions under the (semi)-norm Lip $($.$) and \left(y_{j}\right)_{j} \subset F$ satisfy

where $m \in Æ(X)$. Here

$$
\sup _{\|m\| \leq 1}\left(\sum_{j}\left|\left\langle f_{j}, m\right\rangle\right|^{p^{*}}\right)^{1 / p^{*}}<\infty \text { and }\left\|\left(y_{j}\right)_{j}\right\|_{p}<\infty
$$

$$
\mathcal{N}^{p^{L}}\left(\left(f_{j}\right)_{j},\left(y_{j}\right)_{j}\right)=\sup _{\|m\| \leq 1}\left(\sum_{j}\left|\left\langle f_{j}, m\right\rangle\right|^{p^{*}}\right)^{1 / p^{*}}\left\|\left(y_{j}\right)_{j}\right\|_{p} .
$$

Moreover, $v^{p^{L}}(T):=\inf \mathcal{N}^{p^{L}}\left(\left(f_{j}\right)_{j},\left(y_{j}\right)_{j}\right)$, the infimum being taken over all such representations as above. The collection of all strongly Lipschitz up-nuclear operators from $X$ to $F$ is denoted by $\mathcal{N}^{L}(X, F)$.

Theorem 4.1. $\left(\mathcal{N}^{p^{L}}(X, F), v^{p^{L}}\right)$ is a Banach space of strongly Lipschitz up-nuclear operators.

Proof. (1) It is clear that for any operator $T \in \operatorname{Lip}_{0}(X, F)$ and any scalar $\lambda$, $v^{p^{L}}(T) \geq 0$ and $v^{p^{L}}(\lambda T)=|\lambda| v^{p^{L}}(T)$.

(2) Let $T_{1}, T_{2} \in \operatorname{Lip}_{0}(X, F)$ and $\varepsilon>0$. we can write

$$
T_{i}=\sum_{j} f_{j, i} \otimes y_{j, i}, \quad i=1,2 .
$$

By homogeneity, it is possible to choose representations of $T_{1}$ and $T_{2}$ such that, for $\varepsilon$ given positive real, we have

$$
\begin{aligned}
\left\|\left(y_{j, i}\right)_{j}\right\|_{p} & \leq\left(v^{p^{L}}\left(T_{i}\right)+\varepsilon\right)^{1 / p}, \\
\sup _{\|m\| \leq 1}\left(\sum_{j}\left|\left(\left\langle f_{j, i}, m\right\rangle\right)_{j}\right|^{p^{*}}\right)^{1 / p^{*}} & \leq\left(v^{p^{L}}\left(T_{i}\right)+\varepsilon\right)^{1 / p^{*}},
\end{aligned}
$$

$i=1,2$.

Consequently;

$$
\begin{array}{r}
\left\|\left(y_{j, i}\right)_{j}\right\|_{p} \leq\left(v^{p^{L}}\left(T_{1}\right)+v^{p^{L}}\left(T_{2}\right)+\varepsilon\right)^{1 / p} \\
\sup _{\|m\| \leq 1}\left(\sum_{j}\left|\left(\left\langle f_{j, i}, m\right\rangle\right)_{j, i}\right|^{p^{*}}\right)^{1 / p^{*}} \leq\left(v^{p^{L}}\left(T_{1}\right)+v^{p^{L}}\left(T_{2}\right)+\varepsilon\right)^{1 / p^{*}}
\end{array}
$$

Then

So;

$$
\sup _{\|m\| \leq 1}\left(\sum_{j}\left|\left(\left\langle f_{j, i}, m\right\rangle\right)_{j, i}\right|^{p^{*}}\right)^{1 / p^{*}}\left\|\left(y_{j, i}\right)_{j}\right\|_{p} \leq\left(v^{p^{L}}\left(T_{1}\right)+v^{p^{L}}\left(T_{2}\right)+2 \varepsilon\right) .
$$

$$
v^{p^{L}}\left(T_{1}+T_{2}\right) \leq\left(\nu^{p^{L}}\left(T_{1}\right)+v^{p^{L}}\left(T_{2}\right)+2 \varepsilon\right) .
$$


Proposition 4.1. Let $1<p \leq \infty$, and $T \in \operatorname{Lip}_{0}(X, F)$. Then $T \in \mathcal{N}^{p^{L}}(X, F)$ if, and only if, $T_{L} \in \mathcal{N}^{p}(Æ(X), F)$, with

$$
v^{p^{L}}(T)=v^{p}\left(T_{L}\right)
$$

Proof. It's well known that $\mathcal{N}^{p}(Æ(X), F)$ is a normed operator ideal (see [16]) and $T \in \operatorname{Lip}_{0}(X, F)$, then by [1, Proposition 3.2] we get the equivalence is above with the necessary norm.

Theorem 4.2. $\left(\mathcal{N}^{p^{L}}(X, F), v^{p^{L}}\right)$ is a Banach Lipschitz ideal.

Proof. Since $\mathcal{N}^{p}(Æ(X), F)$ is a normed operator ideal, $T \in \mathcal{N}^{p}(Æ(X), F) \circ \operatorname{Lip}_{0}(X, Æ(X))$ and by [1, Corollary 3.3], $\mathcal{N}^{p^{L}}(X, F)$ is a Banach strongly Lipschitz up-nuclear operator ideal.

\section{Factorization Theorem}

In this section, we characterize operators in $\mathcal{N}^{p^{L}}(X, F)$.

Theorem 5.1. Let $1<p \leq \infty$ and $T \in \operatorname{Lip}_{0}(X, F)$. Then $T \in \mathcal{N}^{p^{L}}(X, F)$ if and only if, $T$ has a factorization $T=a M_{\lambda} B$ such that the following diagram commutes:

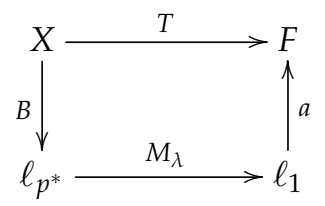

where $B \in \operatorname{Lip}_{0}\left(X, \ell_{p^{*}}\right)$ with $B(0)=0, M_{\lambda} \in \mathcal{L}\left(\ell_{p^{*}}, \ell_{1}\right)$ a diagonal operator and $a \in \mathcal{L}\left(\ell_{1}, F\right)$. Moreover, $v^{p^{L}}(T):=$ $\inf \|a\|\left\|M_{\lambda}\right\| \operatorname{Lip}(B)$, where the infimum is taken over all the above factorizations.

Proof. We know if $T \in \operatorname{Lip}_{0}(X, F)$ there exists a unique linear map $T_{L}: Æ(X) \longrightarrow F$ such that $T=T_{L} \circ \delta_{X}$, more they have the same characteristics. Since $T_{L} \in \mathcal{N}^{p}(Æ(X), F)$, so we have a factorization as following

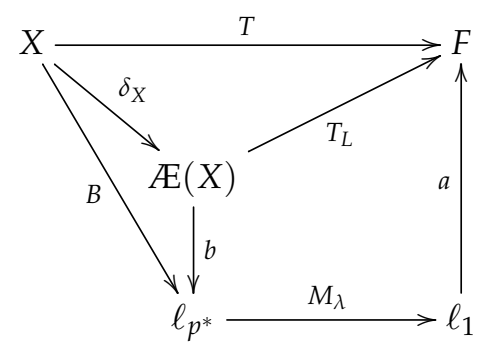

we can see that $T=a M_{\lambda} B$, where $B=b \circ \delta_{X}$.

Conversely, a similar proof as in [4, Theorem 2.2], with

$$
\nu^{p^{L}}(T)=\inf \|a\|\left\|M_{\lambda}\right\| \operatorname{Lip}(B) .
$$


Remark 5.1. For $p=1$, we have $\mathcal{N}^{1}(X, F)=\mathcal{N}_{1}^{L}(X, F)$.

Using Theorem 5.1 we obtain the following results:

Proposition 5.1. Let $1<p \leq q \leq \infty$, then $\mathcal{N}^{p^{L}}(X, F) \subseteq \mathcal{N}^{q^{L}}(X, F)$, with

$$
v^{p^{L}}(T) \geq v^{q^{L}}(T) \text {. }
$$

Proof. We know that a multiplication operator can be factored as (see [17])

$$
M_{\lambda}: \ell_{p^{*}} \stackrel{M_{\alpha}}{\longrightarrow} \ell_{q^{*}} \stackrel{M_{\beta}}{\longrightarrow} \ell_{1}
$$

So,

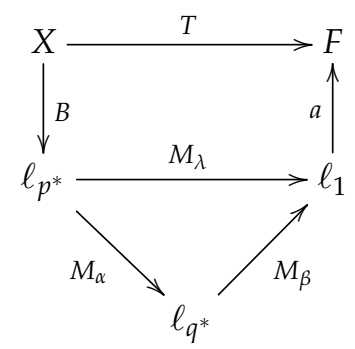

where $M_{\alpha}$ and $M_{\beta}$ are multiplication operators which are given by $\alpha_{n}=\left|\lambda_{n}\right|^{1-\frac{p^{*}}{q^{*}}}$ and $\beta_{n}=\left(\operatorname{sign} \lambda_{n}\right)\left|\lambda_{n}\right|^{\frac{p^{*}}{q^{*}}}(n \in$ $\mathbb{N})$.

$$
\begin{aligned}
v^{p^{L}}(T) & =\inf \operatorname{Lip}(B)\left\|M_{\lambda}\right\|\|a\| \\
& =\inf \operatorname{Lip}(B)\left\|M_{\beta} M_{\alpha}\right\|\|a\| \\
& =\inf \operatorname{Lip}(B)\left\|M_{\alpha}\right\|\left\|M_{\beta}\right\|\|a\| \\
& \geq \inf \operatorname{Lip}(\tilde{B})\left\|M_{\beta}\right\|\|a\| \\
& \geq v^{q^{L}}(T) .
\end{aligned}
$$

Corollary 5.1. Let $1<p \leq \infty$ and $T \in \operatorname{Lip}_{0}(X, F)$. We have

(1) $J_{E} \circ T \in \mathcal{N}^{p^{L}}\left(X, F^{* *}\right) \Leftrightarrow\left(T^{t}\right)^{*} \in \mathcal{N}^{p}\left(\left(X^{\sharp}\right)^{*}, F^{* *}\right)$.

(2) $T \in \mathcal{N}^{p^{L}}(X, F) \Rightarrow\left(T^{t}\right)^{*} \in \mathcal{N}^{p}\left(\left(X^{\sharp}\right)^{*}, F^{* *}\right)$.

Open problem: Under which condition the following implication

$$
J_{E} \circ T \in \mathcal{N}^{p^{L}}\left(X, F^{* *}\right) \Rightarrow T \in \mathcal{N}^{p^{L}}(X, F)
$$

will be true?

\section{Applications}

This section is devoted to some applications such as duality, relationships with known spaces.

Proposition 6.1. If $T \in \mathcal{N}_{p}^{L}(X, F)$, then its transpose $T^{t} \in \mathcal{N}^{p}\left(F^{*}, X^{\sharp}\right)$ and it satisfies

$$
v^{p}\left(T^{t}\right) \leq s v_{p}^{L}(T)
$$


Furthermore, assume $F$ is reflexive. Then, if $T^{t} \in \mathcal{N}^{p}\left(F^{*}, X^{\sharp}\right)$ we have $T \in \mathcal{N}_{p}^{L}(X, F)$, with

$$
v^{p}\left(T^{t}\right)=s v_{p}^{L}(T) .
$$

Proof. By [1, Proposition 2.7, Proposition 3.2], $T \in \mathcal{N}_{p}^{L}(X, F)$ if, and only if, $T_{L} \in \mathcal{N}_{p}(Æ(X), F)$. Then, by [13, Proposition 1], we have $\left(T_{L}\right)^{*} \in \mathcal{N}^{p}\left(F^{*}, Æ(X)^{*}\right)$, wich gives us $T^{t} \in \mathcal{N}^{p}\left(F^{*}, X^{\sharp}\right)$ because

$$
T^{t}: F^{*} \stackrel{\left(T_{L}\right)^{*}}{\longrightarrow} Æ(X)^{*} \stackrel{\delta_{X}^{t}}{\longrightarrow} X^{\sharp} .
$$

Furthermore, as $T^{t}=\delta_{X}^{t} \circ\left(T_{L}\right)^{*}$ so

$$
\begin{aligned}
v^{p}\left(T^{t}\right)=v^{p}\left(\delta_{X}^{t} \circ\left(T_{L}\right)^{*}\right) & \leq v^{p}\left(\left(T_{L}\right)^{*}\right) \\
& \leq v_{p}\left(T_{L}\right) \\
& =s v_{p}^{L}(T)
\end{aligned}
$$

Hence,

$$
\nu^{p}\left(T^{t}\right) \leq s v_{p}^{L}(T)
$$

Conversely, if $T^{t} \in \mathcal{N}^{p}\left(F^{*}, X^{\sharp}\right)$, then $\left(T^{t}\right)^{*} \in \mathcal{N}_{p}\left(\left(X^{\sharp}\right)^{*}, F^{* *}\right)$, therefor $T \in \mathcal{N}_{p}^{L}(X, F)$ because

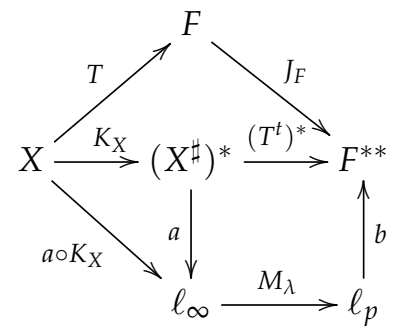

where $K_{X}$ is the evaluation map $K_{X}(x)(f)=f(x), x \in X, f \in X^{\sharp}$ and $J_{E}$ is the canonical injection from $F$ into $F^{* *}$, so,

From (6.1) and (6.2) we get

$$
s v_{p}^{L}(T) \leq v^{p}\left(T^{t}\right)
$$

$$
s v_{p}^{L}(T)=v^{p}\left(T^{t}\right)
$$

Theorem 6.1. If $T \in \mathcal{N}^{p^{L}}(X, F)$, then its transpose $T^{t} \in \mathcal{N}_{p}\left(F^{*}, X^{\sharp}\right)$ and it satisfies

$$
v_{p}\left(T^{t}\right) \leq v^{p^{L}}(T)
$$

Furthermore, assume $F$ is reflexive. Then, if $T^{t} \in \mathcal{N}_{p}\left(F^{*}, X^{\sharp}\right)$ we have $T \in \mathcal{N}^{p^{L}}(X, F)$, with

$$
v_{p}\left(T^{t}\right)=v^{p^{L}}(T) \text {. }
$$

Proof. By Proposition 4.1, $T \in \mathcal{N}^{p^{L}}(X, F)$ if, and only if, $T_{L} \in \mathcal{N}^{p}(Æ(X), F)$. Then, $\left(T_{L}\right)^{*} \in \mathcal{N}_{p}\left(F^{*}, Æ(X)^{*}\right)$, that is to say $T^{t} \in \mathcal{N}_{p}\left(F^{*}, X^{\sharp}\right)$ because

it is clear that $T^{t}=\delta_{X}^{t} \circ\left(T_{L}\right)^{*}$ so

$$
T^{t}: F^{*} \stackrel{\left(T_{L}\right)^{*}}{\longrightarrow} Æ(X)^{*} \stackrel{\delta_{X}^{t}}{\longrightarrow} X^{\sharp},
$$

$$
\begin{aligned}
v_{p}\left(T^{t}\right)=v_{p}\left(\delta_{X}^{t} \circ\left(T_{L}\right)^{*}\right) & \leq v_{p}\left(\left(T_{L}\right)^{*}\right) \\
& \leq v^{p}\left(T_{L}\right) \\
& =v^{p^{L}}(T) .
\end{aligned}
$$


Hence

$$
v_{p}\left(T^{t}\right) \leq v^{p^{L}}(T) .
$$

Conversely, we use the same method used in [13, Proposition 1], if $T^{t} \in \mathcal{N}_{p}\left(F^{*}, X^{\sharp}\right)$ then for any $\varepsilon>0$, it can be written as

$$
T^{t} y^{*}=\sum_{j}\left\langle y^{*}, y_{j}^{* *}\right\rangle f_{j}
$$

for each $y^{*} \in F^{*}$ and $m \in Æ(X)$, with

$$
\sup _{\|m\| \leq 1}\left(\sum_{j}\left|\left\langle f_{j}, m\right\rangle\right|^{p^{*}}\right)^{1 / p^{*}}\left\|\left(y_{j}^{* *}\right)_{j}\right\|_{p} \leq v_{p}\left(T^{t}\right)+\varepsilon
$$

Since $\mathrm{F}$ is reflexive

$$
\sup _{\|m\| \leq 1}\left(\sum_{j}\left|\left\langle f_{j}, m\right\rangle\right|^{p^{*}}\right)^{1 / p^{*}}\left\|\left(y_{j}\right)_{j}\right\|_{p} \leq v_{p}\left(T^{t}\right)+\varepsilon
$$

Hence we have

$$
T=\sum_{j} f_{j} \otimes y_{j}
$$

and (6.5) shows

From (6.3) and (6.6) we get

$$
v^{p^{L}}(T) \leq v_{p}\left(T^{t}\right)
$$

$$
v^{p^{L}}(T)=v_{p}\left(T^{t}\right)
$$

Remark 6.1. Let $T \in \mathcal{N}_{p}^{L}(X, F)^{\text {dual }}$, then by [1, Theorem 3.9] we get $T \in \mathcal{N}_{p}(X, F)^{\text {dual }} \circ$ Lip 0 and by [13, Proposition 1] $T \in \mathcal{N}^{p}(X, F) \circ \operatorname{Lip}_{0}$ so $T \in \mathcal{N}^{p^{L}}(X, F)$.

Proposition 6.2. Let $1<p \leq \infty$ and $T \in \operatorname{Lip}_{0}(X, F)$. If $T \in \mathcal{N}^{p^{L}}(X, F)$, then $T^{t} \in \Pi_{p}\left(F^{*}, X^{\sharp}\right)$, with

$$
\pi_{p}\left(T^{t}\right) \leq v^{p^{L}}(T)
$$

Proof. By Theorem 6.1, $T^{t} \in \mathcal{N}_{p}\left(F^{*}, X^{\sharp}\right)$ if $T \in \mathcal{N}^{p^{L}}(X, F)$. Then by [7, Proposition 5.5, Corollary 5.24], we get $T^{t} \in \Pi_{p}\left(F^{*}, X^{\sharp}\right)$, with

$$
\pi_{p}\left(T^{t}\right) \leq v_{p}\left(T^{t}\right) \leq v^{p^{L}}(T)
$$

Proposition 6.3. Let $1<p \leq \infty$ and $T \in \operatorname{Lip}_{0}(X, F)$. If $T \in \mathcal{N}^{p^{L}}(X, F)$, then $T \in \mathcal{D}_{s t, p^{*}}^{L}(X, F)$, with

$$
d_{s t, p^{*}}^{L}(T) \leq v^{p^{L}}(T) \text {. }
$$

Proof. We have by Proposition 6.2, $T \in \mathcal{N}^{p^{L}}(X, F)$, then $T^{t} \in \Pi_{p}\left(F^{*}, X^{\sharp}\right)$. Hence by [19, Theorem 4.1], $T \in$ $\mathcal{D}_{s t, p^{*}}^{L}(X, F)$, and

$$
d_{s t, p^{*}}^{L}(T)=\pi_{p}\left(T^{t}\right) \leq v^{p^{L}}(T)
$$

We present the previous proposition in another way, using the ideal property.

Proposition 6.4. Let $1<p \leq \infty$ and $T \in \operatorname{Lip}_{0}(X, F)$. If $T \in \mathcal{N}^{p^{L}}(X, F)$, then there exist a Banach space $G, u \in$ $\mathcal{D}_{p^{*}}(G, F)$ and $S \in \operatorname{Lip}_{0}(X, G)$ such that $T=u \circ S$.

Proof. It's direct from Proposition 6.3 and [19, Corollary 3.6].

Acknowledgements. The authors wish to thank Professor Roshdi Khalil for his useful suggestions concerning this paper. 


\section{References}

[1] D. Achour, P. Rueda, E.A. Sánchez-Pérez and R. Yahi, Lipschitz operator ideals and the approximation property, J. Math. Anal. Appl., 436 (2016), 217-236.

[2] R.F. Arens and J. Eells Jr., On embedding uniform and topological spaces, Pacific J. Math., 6 (1956), 397-403.

[3] A. Belacel and D. Chen, Lipschitz $(p, r, s)$-integral operators and Lipschitz $(p, r, s)$-nuclear operators, J. Math. Anal. Appl., 461 (2018), 1115-1137.

[4] D. Chen and B. Zheng, Lipschitz p-integral operators and Lipschitz p-nuclear operators, Nonlinear Anal., 75 (2012), 5270-5282.

[5] J.S. Cohen, Absolutely p-summing, p-nuclear operators and their conjugates, Math. Ann., 201 (1973), 177-200.

[6] A. Defant and K. Floret, Tensor Norms and Operator Ideals, North-Holl. Math. Stud., vol. 176, North-Holland Publishing Co., Amsterdam, (1993).

[7] J. Diestel, H. Jarchow and A. Tonge, Absolutely Summing Operators, Cambridge University Press, (1995).

[8] J.D. Farmer and W.B. Johnson, Lipschitz p-summing operators, Proc. Am. Math.Soc., 137(9)(2009), 2989-2995.

[9] D.J.H. Garling: Inequalities, A journey into Linear Analysis, Cambridge University Press, (2007).

[10] H. Hogbe-Nlend and V. B. Moscatelli, Nuclear and Conuclear Spaces, North- 52. North-Holland Publishing Company, (1981).

[11] R. Khalil, A. Yousef, Isometries of P-nuclear operator spaces, J. Comput. Anal. Appl. 16(2)(2014), 368-374.

[12] J. Lindenstrauss and L. Tzafriri, Classical Banach Spaces, Tome 1. Sequence Springer-Verlag, (1977).

[13] K. Miyazaki, (p,q)-Nuclear and (p,q)-Integral Operators, Hiroshima Math. J., 4 (1974), 99-132.

[14] A. Persson, On some properties of p-nuclear and p-integral operators, Studia Math., (1969), 113-222.

[15] A. Pietsch, Operator ideals, North-Holland Publishing Company, (1980).

[16] O.I. Reinov, On Linear operators with p-nuclear adjoints, Math. FA, (2001).

[17] S. Reisner, A factoriztion theorem in Banach lattices and its application to Lorentz spaces, Annales de l'institut Fourier, tome 31, n 01 , p. (1981), 239-255.

[18] I. Sawashima, Methods of Lipschitz Duals, Lecture Notes in Econom. and Math. Systems, vol. 419, Springer Verlag, pp. (1975), 247-259.

[19] R. Yahi, D. Achour and P. Rueda, Absolutely summing Lipschitz conjugates, Mediterr. J. Math., 13 (2016), 19491961.

[20] N. Weaver, Lipschitz Algebras, World Scientific Publishing Co., Singapore, (1999). 